\title{
Role of afatinib in the treatment of advanced lung squamous cell carcinoma
}

This article was published in the following Dove Press journal:

Clinical Pharmacology:Advances and Applications

Number of times this article has been viewed

\section{Tiziana Vavalà}

ASL CNI, SC Oncologia, Ospedale Civile di Saluzzo, Saluzzo, Cuneo, Italy
Correspondence: Tiziana Vavalà Ospedale Civile di Saluzzo, Via Spielberg

37, 12037 Saluzzo, Cuneo, Italy

Tel +390175215289

Fax +3917521 5668

Email tvavala@hotmail.it
Abstract: Lung cancer treatment has considerably changed over the last few years: the identification of druggable oncogenic alterations and innovative immunotherapic approaches granted lung cancer patients the possibility of more efficient and less toxic therapeutic options than chemotherapy. Nowadays, lung squamous cell carcinomas ( $\mathrm{SqCCs}$ ) patients have the chance to benefit from novel treatment alternatives, including immune checkpoint blockade and antiangiogenic agents and, given positive trial results, from afatinib, a second generation tyrosine kinase inhibitor (TKI) that irreversibly antagonizes ErbB family tyrosine kinase receptors. Considering the role of the ErbB-signaling cascade in lung $\mathrm{SqCC}$, it is relevant to note that ErbB1 (epidermal growth factor receptor [EGFR]) is overexpressed in $85 \%$ of non-small-cell lung carcinomas (NSCLCs), particularly in patients with squamous histology, and is associated with poor prognosis. For this reason, EGFR activity has been investigated as a therapeutic strategy in lung SqCC. Even taking into account statistically positive trial results, anti-EGFR approach still remains controversial in unselected/wild-type EGFR lung SqCC patients, as well as the optimal timing and sequencing of all available targeted therapies considering the approval of immunotherapeutic agents. This review analyzes current data about EGFR inhibition in lung SqCC with a specific focus on afatinib in order to elucidate available clinical evidence supporting EGFR targeting in this setting as well as a future management of advanced lung SqCCs in the context of new emerging immunotherapeutic drugs.

Keywords: lung cancer, NSCLC, tyrosine kinase inhibitor, LUX-Lung, EGFR, TKI

\section{Introduction}

Lung cancer is the most frequently diagnosed type of tumor and the leading cause of cancer-related death worldwide with an estimated 1.8 million new lung cancer cases and 1.59 million deaths in 2012, according to the last GLOBOCAN estimates of cancer incidence and mortality produced by the International Agency for Research on Cancer (IARC). ${ }^{1}$

Lung squamous cell carcinoma (SqCC) is the second most common histology in non-small-cell lung carcinomas (NSCLCs) accounting for 20-30\% of NSCLC cases and, as the majority of lung cancer patients, presents often with advanced stage of the disease when diagnosed. ${ }^{2}$

Compared to the most frequent advanced lung adenocarcinoma, for which targeted therapies are now available if they present actionable mutations, treatment options for advanced lung SqCC, both in first-line and relapsed/refractory settings, have been, for a long time, a relevant unmet medical need. Extensive research was then addressed toward novel treatment strategies, and, in the last few years, several new 
drugs have been approved for squamous histology: these include anti-epidermal growth factor receptor (EGFR) monoclonal antibodies (mAbs) (necitumumab and cetuximab) in combination with standard frontline chemotherapy and immune-checkpoint inhibitors such as nivolumab, pembrolizumab, atezolizumab, the anti-vascular endothelial growth factor-2 (VEGFR-2) antibody ramucirumab (combined with docetaxel), and the ErbB-family blocker, afatinib. ${ }^{3}$

Considering EGFR pathway, the biological mechanism accounting for the efficacy of EGFR inhibition in lung SqCC is probably multifactorial: in two-thirds of lung SqCCs with activation of the phosphatidylinositol 3-kinase (PI3K)/receptor tyrosine kinase/Ras pathways, recent genomic analyses suggest that EGFR alterations occur with a frequency of $\sim 9 \%$ and second-generation EGFR tyrosine kinase inhibitors (TKIs), such as afatinib, which irreversibly bind to enzymatically active ErbB receptors, enable sustained ErbB family blockade including receptor activation, dimerization, and resistance inhibition, explaining their enhanced efficacy over erlotinib in lung SqCC. Preclinical data suggested that lung SqCC pathobiology has a strong dependency from the ErbB family pathway: ErbB1 (EGFR) is overexpressed in $85 \%$ of NSCLCs, particularly in patients with squamous histology $(\sim 60-80 \%)$ and is associated with poor prognosis; HER2 and HER3 are overexpressed in $20-30 \%$ of lung SqCCs overall and present genetic aberrations in $\sim 3$ and $4 \%$, respectively; finally, some lung SqCCs (7-10\%) also demonstrated EGFR gene copy-number alterations, providing a biological rationale for using EGFR-targeted agents as a treatment option in this setting. ${ }^{4-7}$

However, it is still unclear if high levels of EGFR expression in lung SqCC present the same meaningful clinical impact as EGFR sensitizing mutations in adenocarcinoma patients. As a consequence, even taking into account statistically positive trial results in squamous histology, EGFR inhibition in unselected/wild-type EGFR lung SqCC cases still remains controversial. ${ }^{8}$ Probably, in addition to EGFR, further members of the ErbB family play a role in the pathogenesis of squamous disease as well as genetic aberrations in numerous intracellular downstream signaling networks of the ErbB receptors, such as Kirsten rat sarcoma virus GTPase (KRAS, 3\%), Harvey rat sarcoma virus GTPase (HRAS, $3 \%$ ), Braf serine/threonine-protein kinase (BRAF, 4\%), and neurofibromin 1 (NF1, 11\%). ${ }^{9}$ Other studies have identified HER4 mutations in lung SqCCs, and another one demonstrated that genomic aberrations in neuregulin 1 (NRG1), one of the similar ligands of the ErbB family receptors, are a recurrent feature of lung SqCCs. ${ }^{10-14}$ Even if controversial,
anti-EGFR strategy in squamous histology needs to be further elucidated considering also that lung SqCCs share several molecular alterations with head and neck SqCC (not human papilloma virus associated), in which, particularly EGFR, is not only an independent prognostic factor of outcome but also a first-choice therapeutic target. ${ }^{15}$

The aim of this review is to analyze current data about EGFR inhibition as a therapeutic strategy in lung SqCC with a specific focus on afatinib. A summary of evidence from randomized trials of afatinib will also be presented in order to discuss about rationale and available clinical evidence supporting the targeting of EGFR in this setting as well as future management of advanced lung SqCC contextualized with the recent approval of immunotherapy approaches.

\section{Overview of current treatments for lung SqCC}

EGFR pathway inhibition has been investigated as a therapeutic strategy in NSCLC using both small molecules, EGFR TKIs and anti-EGFR mAbs. Both classes of drugs have been shown to decrease signal transduction of the EGFR pathway, through different mechanisms of action. ${ }^{16,17}$ Mutations activating EGFR signaling pathway, like L858R mutation in exon 21 and exon 19 deletions, are used as biomarkers to preferentially identify NSCLC patients suitable for EGFR TKI therapy, but clinical responses in NSCLC patients with lack of EGFR mutations have been described in retrospective trials suggesting that a mutation-independent mechanism could potentially contribute to the observed efficacy of EGFR TKI therapies. ${ }^{18-20}$

Based on this rationale and, as previously described, considering frequent EGFR overexpression and EGFR gene copy-number alterations in lung SqCC, different studies assessed EGFR-targeted agents in this setting of patients both in first and subsequent lines of therapy.

\section{First-line approaches for lung SqCC}

Anti-EGFR mAbs were evaluated as monotherapy in frontline management of patients with advanced NSCLC evidencing a limited single-agent activity (response rate $\sim 4 \%$ ); as a consequence researchers preferred to move to analysis of anti-EGFR mAbs in combination with cytotoxic therapies. ${ }^{21}$

Phase III SQUIRE trial evaluated necitumumab, a secondgeneration IgG1 anti-EGFR recombinant fully human $\mathrm{mAb}$, in combination with gemcitabine/cisplatin versus chemotherapy alone in 1,093 lung SqCC patients. Addition of necitumumab significantly improved overall survival (OS) versus chemotherapy alone (11.5 versus 9.9 months, hazard 
ratio [HR] 0.84, 95\% confidence interval [CI] 0.74-0.96; $P=0.01)$. However, improved efficacy needs to be considered in the context of additional toxicity as in the necitumumab plus gemcitabine/cisplatin arm, the number of patients with at least one grade 3 or worse adverse event (AE) was higher $(\mathrm{n}=388,72 \%$ of 538 patients) than in the chemotherapy $\operatorname{arm}(n=333,62 \%$ of 541$)$, as was the incidence of serious AEs ( $n=257,48 \%$ of 538 versus $203,38 \%$ of 541 patients) and treatment discontinuations due to AEs (31.2\% versus $24.6 \%$ ) in the combination arm. ${ }^{22}$

On the contrary, cetuximab, a chimeric IgG1 mAb, was not studied in Phase III trials specifically dedicated to lung SqCC patients in association with chemotherapy. However, in a Phase III study, the FLEX trial (which enrolled histologically unselected patients), a superior OS (12.0 versus 9.6 months) was described with cetuximab for patients with high EGFR expression levels; particularly, among patients with high EGFR expression those with squamous histology were found to benefit the most from cetuximab (11.2 versus 8.9 months, HR: 0.62, 95\% CI 0.43-0.88). ${ }^{23}$ Nevertheless, it is important to note that in a prespecified analysis of the SQUIRE trial, high levels of EGFR expression did not predict improved OS with necitumumab. ${ }^{22,24}$

In contrast to anti-EGFR antibodies, first-line EGFR TKIs in combination with chemotherapy failed to demonstrate a clinical benefit over chemotherapy alone in patients with squamous but also nonsquamous lung carcinoma. ${ }^{17,25-27}$ First-line EGFR TKI monotherapy was also evaluated, and considering several prospective trials that confirmed EGFR TKIs inferiority compared to chemotherapy in unselected or EGFR wild-type NSCLC patients, it is not recommended, at the present time, in lung SqCC without known EGFR mutations. ${ }^{17}$

The development of novel treatment strategies for lung SqCC patients expanded therapeutic options in the frontline setting as demonstrated by immunocheckpoint inhibitors.

Reck et al evaluated pembrolizumab, a humanized mAb against programmed death-1 (PD-1) as first-line therapy for metastatic NSCLC compared to different chemotherapy regimens in the Phase III KEYNOTE-024 trial. ${ }^{28}$ Previously, untreated advanced NSCLC patients $(n=305, n=56$ with squamous histology) with programmed death-ligand 1 (PD-L1) expression, at least $50 \%$ of tumor cells, and no EGFR sensitizing mutation or anaplastic lymphoma kinase (ALK) rearrangements, were randomly assigned to receive either pembrolizumab (at a fixed dose of $200 \mathrm{mg}$ every 3 weeks) or platinum-based chemotherapy as per investigator's choice. Progression-free survival (PFS) was the primary end-point and was significantly longer in the pembrolizumab arm compared to the chemotherapy arm (10.3 versus 6 months; HR $0.50 ; P<0.001$ and HR of $0.35 ; 95 \%$ CI, $0.17-0.71$ for squamous histology, even considering the limited number of patients). OS was also significantly better in the pembrolizumab group (HR 0.60; 95\% CI, 0.41-0.89; $P=0.005$ ). ${ }^{28}$

Based on significant improvements in PFS and OS reported by this study, US Food and Drug Administration (FDA) approved pembrolizumab for first-line treatment of metastatic NSCLC patients, with no EGFR or ALK genomic tumor aberrations and whose tumors have high PD-L1 expression (tumor proportion score [TPS] of $\geq 50 \%$ ) as determined by an FDA-approved test. ${ }^{29}$

However, even considering relevant results of KEYNOTE-024 trial, it is important to report that previously, a similar study, CHECKMATE-026, a phase III trial investigating nivolumab (a PD-1 checkpoint-inhibitor) in a front-line setting, in stage IV or recurrent untreated NSCLC with PD-L1 tumor-expression level of $1 \%$, did not show a significantly longer PFS from nivolumab compared to chemotherapy. ${ }^{30}$

Finally, EGFR TKIs administration as long-term maintenance therapy was supported by preclinical data, suggesting a relationship between cytotoxic resistance and activation of the EGFR pathway. ${ }^{31}$ To date, SATURN has been the largest Phase III trial to assess erlotinib as maintenance therapy in unselected NSCLC patients with stable disease or partial/ complete response after induction chemotherapy. Particularly, erlotinib was found to delay disease progression in patients with squamous histology $(n=360 / 889)$, irrespective of response to induction chemotherapy (PFS: HR 0.76, 95\% CI 0.60-0.95). Among patients with stable disease, the greatest OS benefit was in lung SqCC patients (OS: HR 0.67, 95\% CI 0.48-0.92). ${ }^{32}$

\section{Second-line approaches and beyond for lung $\mathrm{SqCC}$}

For many years, docetaxel and erlotinib remained the only standard second-line choices for lung SqCC treatment until, in December 2014, the FDA approved the anti-VEGFR-2 antibody ramucirumab in combination with docetaxel, for the treatment of metastatic NSCLC patients who progressed during or after a platinum-based chemotherapy. ${ }^{3}$ Nivolumab and atezolizumab were then approved in March 2015 and October 2016, respectively, for the treatment of metastatic NSCLC patients, progressing during or after a platinumbased chemotherapy, pembrolizumab in October 2015, for metastatic NSCLC patients whose tumors expressed PD-L1 (evaluated with the approved companion diagnostic). 
Finally, afatinib (an EGFR TKI) was approved in April 2016, for the treatment of patients with metastatic lung SqCC progressing after a platinum-based chemotherapy. ${ }^{3,33-35}$ In the Phase III REVEL study, NSCLC patients were treated with docetaxel with or without ramucirumab: an OS benefit was evidenced with ramucirumab/docetaxel in the entire population (10.5 versus 9.1 months, HR 0.86 , 95\% CI $0.75-0.98, P=0.023)$. In those patients treated with ramucirumab/docetaxel with squamous cell histology (25\%), the OS benefit was 9.5 versus 8.2 months (HR: 0.88 , 95\% CI 0.69-1.13) in the placebo-docetaxel subgroup; however, it is essential to note that subgroup analyses in this study were not preplanned. ${ }^{33,36}$

In CHECKMATE-017 study, nivolumab was compared with docetaxel in 272 advanced, pretreated, lung SqCC patients (PD-L1 positivity was not a requirement). The trial demonstrated an advantage of nivolumab versus docetaxel with a median OS of 9.2 months versus 6 months (HR 0.59, CI $0.44-0.79, P=0.001$ ) and overall response rate (ORR) $20 \%$ versus $9 \%$ ( $P=0.008)$, respectively, leading to the first FDA approval for immunotherapy in NSCLC. ${ }^{37}$

KEYNOTE-010, a randomized Phase III trial analogous to CHECKMATE-017 and -057, compared pembrolizumab at two doses, 2 and $10 \mathrm{mg} / \mathrm{kg}$ every 3 weeks, with docetaxel in 1,034 NSCLC patients. Only patients with at least 1\% PDL1-positive staining were enrolled, with the last 593 patients stratified by PD-L1 positivity using a $50 \%$ cutoff. Patients treated with either dose of pembrolizumab had a higher median OS than with docetaxel ( $10 \mathrm{mg} / \mathrm{kg}: 12.7$ versus 8.5 months; HR $0.61, P<0.0001 ; 2 \mathrm{mg} / \mathrm{kg}: 10.4$ months, HR 0.71, $P=0.0008)$. In the subgroup analysis, both lung SqCC and non-SqCC favored pembrolizumab, consistent with results from nivolumab clinical trials. ${ }^{38}$ Finally, atezolizumab is an IgG1 mAb against PD-L1, fully humanized. It was evaluated in the OAK trial, a Phase III study enrolling pretreated NSCLC patients who were randomly assigned to receive either atezolizumab $(n=425)$ or docetaxel $(n=425)$. The median OS was 13.8 compared to 9.6 months in the atezolizumab arm versus docetaxel arm (HR 0.73, 95\% CI 0.62-0.87, $P=0.0003$ ). OS improvement was comparable in patients with squamous (HR 0.73, 95\% CI $0.54-0.98, n=112$ in the atezolizumab group and $n=110$ in the docetaxel group) or nonsquamous (HR 0.73, 95\% CI $0.60-0.89, n=313$ and $n=315$ ) histology. ${ }^{39}$

\section{Afatinib - mechanism of action and selected studies}

Afatinib (BIBW 2992) is an irreversible blocker of EGFR, HER2, and inhibits signaling from all ErbB hetero- and homodimers. ${ }^{40,41}$
It was originally developed with the aim to improve clinical outcomes versus first-generation EGFR inhibitors as, in preclinical studies, it was evidenced to be more effective in suppressing tyrosine kinase activity of both wild-type and activated EGFR or HER2 mutants in lung cancer cell lines; these afatinib-sensitive cancer cell lines contained erlotinib-resistant isoforms, wild-type EGFR, L858R/T790M double mutation, or HER2 overexpression and paved the way for Phase I studies. ${ }^{11,41,42}$

The largest clinical trials focusing on afatinib were the LUX-Lung studies 1-8, sponsored by Boehringer Ingelheim. Of these, LUX-Lung 1-4 and LUX-Lung 6 and 7 were addressed specifically to adenocarcinoma histology while LUX-Lung 5 and 8 were specifically addressed to NSCLC (unselected histology) or lung SqCC (Table 1). LUX-Lung 1 was a Phase IIB/III trial enrolling 585 stage IIIB/IV patients with adenocarcinoma histology who had received one or two previous chemotherapy regimens and presented progressive disease after at least 12 weeks of treatment with erlotinib or gefitinib. In this study, afatinib (50 mg/day) plus best supportive care (BSC) was compared to placebo plus BSC. This trial did not require EGFR mutations; however, they were present in $96(68 \%)$ patients. The primary end-point was OS, analyzed on an intention-to-treat basis. The median OS was 10.8 months (95\% CI 10.0-12.0) in the afatinib group and 12 months (10.2-14.3) in the placebo group (HR 1.08, 95\% CI $0.86-1.35 ; P=0.74)$. The lack of a significant difference in OS between the two groups was hypothesized to be the result of subsequent different treatments; in fact, 257 (68\%) patients in the afatinib group and $153(79 \%)$ patients in the placebo group underwent further therapies. In this trial, afatinib induced AEs such as diarrhea $(339,87 \%$ of 390 patients; $66.1 \%$ were grade 3$)$ and rash or acne $(305,78 \%$ patients; $56.1 \%$ were grade 3 ), while the same events occurred less often in the placebo group (18, 9\% of 195 patients had diarrhea; $31,16 \%$ had rash or acne, all being grade 1 or 2 ). Drug-related serious AEs occurred in $39(10 \%)$ patients in the afatinib group and one $(<1 \%)$ patient in the placebo group. ${ }^{43}$

LUX-Lung 2 was a Phase II single-arm study enrolling 129 stage IIIB/IV EGFR mutated patients with adenocarcinoma histology who were inhibitor naive and had no more than one previous chemotherapy regimen for advanced disease. Afatinib at starting doses of $50 \mathrm{mg}$ daily or $40 \mathrm{mg}$ daily was proposed in order to establish whether tolerability could be improved with preservation of antitumor activity. The primary end-point was the percentage of patients with a confirmed overall response (OR, complete response or partial response) on the basis of Response Evaluation Criteria in Solid Tumors 1.0 (RECIST 1.0, independent review). Of all patients, 99 
Table I Afatinib evaluations in lung SqCC in cited studies

\begin{tabular}{|c|c|c|c|c|c|c|c|}
\hline Study & Design & $\begin{array}{l}\text { Patients } \\
\text { (n) }\end{array}$ & Histology & $\begin{array}{l}\text { EGFR } \\
\text { mutation }\end{array}$ & $\begin{array}{l}\text { Afatinib } \\
\text { dose }\end{array}$ & $\begin{array}{l}\text { Control } \\
\text { arm }\end{array}$ & Results \\
\hline LUX-Lung $5^{48}$ & Phase III & $\mathrm{I}, 154$ & $\begin{array}{l}\text { Adenocarcinoma/ } \\
\text { squamous cell } \\
\text { carcinoma }\end{array}$ & $\begin{array}{l}\text { Not } \\
\text { required }\end{array}$ & $\begin{array}{l}\text { Part A: } \\
50 \mathrm{mg} / \mathrm{d} \\
\text { Part B: } \\
40 \mathrm{mg} / \mathrm{d}+ \\
\text { paclitaxel }\end{array}$ & $\begin{array}{l}\text { Part A: none } \\
\text { Part B: } \\
\text { investigator's } \\
\text { choice }\end{array}$ & $\begin{array}{l}\text { PFS: } 5.6 \text { versus } 2.8 \text { months }(P=0.003) \\
\text { (analysis of } 202 \text { patients) }\end{array}$ \\
\hline LUX-Lung $8^{35}$ & Phase III & 795 & $\begin{array}{l}\text { Squamous cell } \\
\text { carcinoma }\end{array}$ & $\begin{array}{l}\text { Not } \\
\text { required }\end{array}$ & $40 \mathrm{mg} / \mathrm{d}$ & Erlotinib & PFS: 2.4 versus 1.9 months $(P=0.042)$ \\
\hline $\begin{array}{l}\text { LUX-Lung } \\
8, \text { VeriStrat } \\
\text { evaluation }{ }^{7}\end{array}$ & Retrospective & 795 & $\begin{array}{l}\text { Squamous cell } \\
\text { carcinoma }\end{array}$ & $\begin{array}{l}\text { Not } \\
\text { required }\end{array}$ & $40 \mathrm{mg} / \mathrm{d}$ & Erlotinib & $\begin{array}{l}675 \text { were classified: VS-G: } 4 \text { I } 2 \text {, VS-P: } \\
263 \\
\text { OS in VS-G } \\
\text { Afatinib versus erlotinib: HR } 0.79,95 \% \\
\text { Cl: } 0.63-0.98 \\
\text { OS in VS-P } \\
\text { Afatinib versus erlotinib: HR } 0.90,95 \% \\
\text { CI } 0.70-1.16 \\
\text { OS in VS-G versus VS-P patients in } \\
\text { overall VS-classified population: HR } \\
0.4 I, 95 \% \text { Cl } 0.35-0.49\end{array}$ \\
\hline $\begin{array}{l}\text { LUX-Lung 8, } \\
\text { symptoms } \\
\text { and QoL }{ }^{61}\end{array}$ & Prospective & 795 & $\begin{array}{l}\text { Squamous cell } \\
\text { carcinoma }\end{array}$ & $\begin{array}{l}\text { Not } \\
\text { required }\end{array}$ & $40 \mathrm{mg} / \mathrm{d}$ & Erlotinib & $\begin{array}{l}\text { Questionnaire compliance: } \\
\text { Afatinib, } 77.3-99 \% \\
\text { Erlotinib, } 68.7-99 \% \\
\text { Improved scores for GHS/QoL } \\
(P=0.04 \mathrm{I}) \text { : } \\
\text { Afatinib: } 36 \% \\
\text { Erlotinib: } 28 \% \text {; } \\
\text { Improved scores for cough }(P=0.029) \\
\text { Afatinib: } 43 \% \\
\text { Erlotinib } 35 \%\end{array}$ \\
\hline
\end{tabular}

Abbreviations: $\mathrm{Cl}$, confidence interval; EGFR, epidermal growth factor receptor; GHS, global health status; HR, hazard ratio; OS, overall survival; PFS, progression-free survival; QoL, quality of life; SqCC, squamous cell carcinoma; VS-G, VeriStrat-good; VS-P, VeriStrat-poor.

patients received afatinib $50 \mathrm{mg}$ daily and 30 patients received afatinib $40 \mathrm{mg}$ daily. Of the whole population, 79 (61\%) of 129 patients presented an OR (two complete responses, 77 partial responses). When analyzed by starting dose, analogous proportions of patients had similar ORs (61, 62\% of 99 patients at $50 \mathrm{mg}$ versus $18,60 \%$ of 30 patients at $40 \mathrm{mg}$ ). Even if no differences were found in terms of response rates between the two dosing groups, the most common toxicities of afatinib were higher in those patients who received $50 \mathrm{mg}$ daily: 22 , $22 \%$ of 99 patients receiving afatinib $50 \mathrm{mg}$ as starting dose, presented diarrhea grade 3 and $28,28 \%$ of 99 patients, had rash or acne, while two, $7 \%$ of 30 patients receiving afatinib $40 \mathrm{mg}$ as starting dose, evidenced both diarrhea and rash or acne, grade 3. Treatment-related serious AEs were also more common in patients receiving a $50 \mathrm{mg}$ starting dose (14 of 99 patients versus two of 30 patients). Because of this benefit, the dose of $40 \mathrm{mg} /$ day of afatinib was chosen for subsequent Phase III trials. ${ }^{44}$

LUX-Lung 3 and LUX-Lung 6 were Phase III randomized trials involving EGFR-mutated, stage IIIB/IV lung cancer patients with adenocarcinoma histology who underwent up to six cycles of platinum doublet chemotherapy as first-line treatment. In LUX-Lung 3 study, 345 patients, from all over the world, were included and afatinib $40 \mathrm{mg}$ /day was compared with pemetrexed/cisplatin, while in LUX-Lung 6, 364 patients from East Asia were evaluated and gemcitabine/ cisplatin was the control arm. PFS was the primary end-point for both studies and assessed by independent review, while secondary end-points included OS. LUX-Lung 3 presented an advantage in terms of PFS of 11.1 versus 6.9 months (HR $0.58,95 \%$ CI $0.43-0.78, P=0.001$ ) when afatinib was compared with the control arm. In LUX-Lung 6, afatinib presented a PFS of 11 versus 5.6 months for the platinumbased chemotherapy arm (HR $0.28, P=0.0001$ ). However, both trials failed to show a benefit in OS. ${ }^{45,46}$

LUX-Lung 4 was a Japanese single-arm Phase II study, enrolling a total of 62 stage IIIB/IV adenocarcinoma patients whose disease progressed after at least 12 weeks of prior gefitinib and/or erlotinib. EGFR mutation in their primary tumor was positive in 45 (72.6\%) of 62 treated patients, according 
to local and/or central laboratory analyses, and 51 patients (82.3\%) presented acquired resistance to erlotinib and/or gefitinib. Patients received afatinib $50 \mathrm{mg} /$ day. The primary end-point was OR (complete response or partial response) by independent review, which was confirmed (partial response) in five $(8.2 \% ; 95 \%$ CI, $2.7-18.1)$ of 61 evaluable patients. The most common afatinib-related AEs were diarrhea (100\%) and rash/acne (91.9\%). Treatment-related AEs leading to afatinib discontinuation occurred in 18 patients (29\%), of whom four patients also had progressive disease. ${ }^{47}$

LUX-Lung 5 study was a randomized Phase III trial, which assessed, between April 2010 and May 2011, the benefit of afatinib in combination with chemotherapy enrolling 1,154 NSCLC patients ( $\mathrm{n}=90,7.8 \%$ had squamous histology) who had previously been treated with chemotherapy, and/or a first-generation EGFR inhibitor (gefitinib/erlotinib). ${ }^{48}$ This study was divided into two parts: in part A, all patients were initially treated with $50 \mathrm{mg}$ of afatinib per day until progressive disease. Those who received afatinib for 12 weeks or longer were screened $(n=223)$ and randomized $(n=202$, $\mathrm{n}=17$ had squamous histology) 2:1 into part B: afatinib/ paclitaxel combination ( $40 \mathrm{mg} /$ day; $80 \mathrm{mg} / \mathrm{m}^{2}$ per week) or mono-chemotherapy alone (chemotherapy agent according to investigator's choice). In 202 patients analyzed in 2014, the combination treatment arm showed an increased PFS when compared with chemotherapy alone (5.6 versus 2.8 months; HR $0.60,95 \%$ CI $0.43-0.85, P=0.003)$. Objective response rate (RR) was higher in the combination arm (32.1 versus 13.2\%, $P=0.005) .{ }^{11}$ Among 90 patients with lung SqCC (never smokers: $24 \%$ ), the median PFS and ORR were 3.7 months and $6 \%$, respectively; 17 (8.4\%) of them were randomized (2:1) into part B of the trial: afatinib/paclitaxel $(n=11)$ or chemotherapy alone $(n=6)$ arm. The median PFS was 8.8 months in the afatinib/paclitaxel group compared with 1.9 months in the investigator's choice group (HR $0.15,95 \%$ CI $0.03-0.62, P=0.003) .{ }^{49}$ Concerning toxicities of this last group of patients, these will be discussed in the next paragraph. Considering these data and the limited number of patients with lung SqCC involved, any convincing conclusions, particularly for squamous histology, could not be drawn. ${ }^{11}$

LUX-Lung 7 was a Phase IIB study involving 319 EGFRmutated, stage IIIB/IV NSCLC patients (99\% with adenocarcinoma and $1 \%$ with mixed histology, respectively). They were randomly assigned to receive afatinib (40 mg/day) or gefitinib (standard dose of $250 \mathrm{mg}$ daily), which were compared as first-line treatment. Co-primary end-points were PFS by independent central review, time-to-treatment failure, and
OS. The median PFS was 11 months (95\% CI 10.6-12.9) with afatinib versus 10.9 months ( $95 \%$ CI 9.1-11.5) with gefitinib, and the median time-to-treatment failure was 13.7 months (95\% CI 11.9-15) with afatinib versus 11.5 months (95\% CI 10.1-13.1) with gefitinib. OS data were published in a subsequent article: after a median follow-up of 42.6 months, the median OS was 27.9 for the afatinib arm versus 24.5 months (HR 0.86, 95\% CI 0.66-1.12, $P=0.258$ ) for the gefitinib arm. Prespecified subgroup analyses showed similar OS trends in the afatinib versus gefitinib group; particularly, they showed 30.7 versus 26.4 months (HR $0.83,95 \%$ CI $0.58-1.17, P=0.284$ ) in patients with exon 19 deletion, in the two arms, respectively, and 25 versus 21.2 months (HR $0.91,95 \%$ CI $0.62-1.36, P=0.658)$ in patients with L $858 \mathrm{R}$ mutations. Most patients (afatinib, 72.6\%; gefitinib, 76.8\%) had at least one subsequent systemic anticancer treatment following discontinuation of afatinib/gefitinib; 20 (13.7\%) and $23(15.2 \%)$ patients received a third-generation EGFR TKI. The most common treatment-related grade 3 and 4 AEs were diarrhea ( $20.1 \%$ of 160 patients given afatinib versus $2,1 \%$ of 159 given gefitinib), rash or acne (15.9\% patients given afatinib versus 5, 3\% of those given gefitinib) and liver enzyme elevations (no patients given afatinib versus $14.9 \%$ of those given gefitinib). Serious treatment-related AEs occurred in 17 (11\%) patients in the afatinib group and seven (4\%) in the gefitinib group. Ten $(6 \%)$ patients in each group discontinued treatment due to drug-related AEs. In the afatinib group, 15 (9\%) fatal AEs occurred versus 10 $(6 \%)$ in the gefitinib group. All but one of these deaths were considered unrelated to treatment; one patient in the gefitinib group died from drug-related hepatic and renal failure. ${ }^{50,51}$

\section{Afatinib in LUX-Lung 8 versus erlotinib in lung $\mathrm{SqCC}$}

Among LUX-Lung trials, LUX-Lung 8 was the only study focused on lung SqCC and the largest Phase III trial in second-line treatment for this histology. ${ }^{35}$ Patients were not preselected for the presence of EGFR mutational status at baseline, as testing for EGFR is not standard practice for lung SqCCs. The rationale was based on the hypothesis that even if EGFR mutations are rarely found in lung SqCC, the inhibition of disease progression by TKIs may not be performed exclusively by mutated EGFRs but through different molecular mechanisms, since overexpression levels of wildtype EGFR are higher in this histology. ${ }^{4}$ Authors compared afatinib ( $40 \mathrm{mg} /$ day, $\mathrm{n}=398$ patients) versus erlotinib ( $150 \mathrm{mg}$ / day, $\mathrm{n}=397$ patients) in stage IIIB/IV squamous patients only (32, 8\% with mixed histology) who had progressed after at 
least four cycles of chemotherapy with platinum doublet agents. Primary end-point was PFS. At the time of the primary analysis (median follow-up 18.4 months), the median PFS was 2.4 versus 1.9 months (HR 0.82, 95\% CI 0.68-1.00, $P=0.042$ ), median OS was slightly better in the afatinib arm than in the erlotinib one: 7.9 versus 6.8 months (HR 0.81 , 95\% CI 0.69-0.95, $P=0.007$ ), while the disease control rate (DCR) was $51 \%$ ( $n=201 / 398$ patients) versus $40 \%$ ( $n=157 / 397$ patients), $P=0.002$, even though percentage of subjects with an OR did not show any significant difference between the two groups (22.6 versus $11.3 \%, P=0.055)$. The AE profile of afatinib in LUX-Lung 8 was consistent with previous studies and is detailed in the next paragraph. ${ }^{35}$ On the basis of LUXLung 8 results, on April 2016, the FDA approved afatinib as a new oral treatment option for patients with lung SqCC whose disease progressed after treatment with platinum-based chemotherapy. ${ }^{52}$ However, it is important to highlight that previously, the TAILOR trial by Garassino et al and DELTA study by Kawaguchi et al did not evidence superiority of EGFR TKIs over chemotherapy in the treatment of advanced NSCLC (unselected and EGFR wild-type patients). ${ }^{53,54}$ Additionally, docetaxel was more effective than erlotinib for EGFR wildtype NSCLC in the TAILOR study, with slightly improved PFS: 2.9 with docetaxel versus 2.4 months with erlotinib (adjusted HR $0.71,95 \%$ CI, 0.53-0.95; $P=0.02$ ); and median OS: 8.2 months for docetaxel versus 5.4 months for erlotinib (HR 0.73, 95\% CI, 0.53-1.00; $P=0.05$ ). As a consequence, it may have been preferable to compare docetaxel as the control arm, instead of erlotinib, in the LUX-Lung 8 trial; besides, 1.1 month difference in OS in a head-to-head comparison of afatinib versus erlotinib is clinically questionable particularly regarding its translation into routine clinical practice, considering the higher risk of toxicity. ${ }^{55}$

In any case, it is clear that deepening knowledge of the EGFR pathway in lung SqCC could be a major issue particularly considering the wide mechanism of action of afatinib. To date, two studies illustrated afatinib activity in advanced NSCLC patients who were lacking EGFR mutations but presented an increased EGFR gene copy number by amplification or polysomy. ${ }^{56,57}$ Specifically, Cappuzzo et al investigated the activity and safety of afatinib in advanced NSCLC with increased EGFR gene copy number and/or gene amplification by fluorescence in situ hybridization (FISH), with or without EGFR mutation in a Phase II study. Of the 223 patients screened, 69 patients were FISH positive (patients with high polysomy or gene amplification were considered FISH positive). The ORR was $13 \%$ in the whole population $(n=9 / 69$ patients). Higher ORRs were observed in patients with gene amplification ( $20 \% ; n=5 / 25$ patients) and in those with EGFR mutation-positive tumors ( $25 \% ; n=3 / 12$ patients). DCR was $50.7 \%$ overall $(n=35 / 69$ patients; median duration: 24.9 weeks) with higher DCRs evidenced in those subjects with gene amplification, 64\% ( $n=16 / 25$ patients), and in those with EGFR mutation-positive tumors, $66.7 \%(n=8 / 12$ patients) ${ }^{57}$ However, in a post hoc analysis of LUX-Lung 8 , results were somewhat different: a subgroup of 238 patients was selected and divided into presumed treatment benefit group (PFS >2 months; $\mathrm{n}=144$ ) and treatment-refractory group (PFS $<2$ months; $n=94$ ). The percentage of EGFR mutated patients was low $(14,6 \%)$ and only 15 patients had EGFR amplification ( $6 \%$, nine in the afatinib group and six in the erlotinib group). Hence, the improvement of survival outcomes with afatinib in this study was probably not driven by EGFR molecular aberrations and might have been a consequence of afatinib irreversible inhibition of ErbB. ${ }^{11,35}$

Clinical efficacy of afatinib in EGFR wild-type patients may be due to compensatory signaling suppression through other ErbB family members. ${ }^{55}$ These data implied that afatinib inactivates multiple aberrant signaling cascades downstream of ErbB receptors probably through inhibition of receptor dimerization in squamous histology. ${ }^{58}$ Another possible mechanism of afatinib was evidenced in $\mathrm{H} 358$ and H441 NSCLC cell lines, which lack EGFR mutations. The apoptotic effect of afatinib on cancer cells is associated with downregulation of cancerous inhibitor of protein phosphatase 2A (CIP2A), upregulation of protein phosphatase 2A (PP2A) activity, and reduction in serine/threonine kinase 1 (AKT) phosphorylation. Afatinib downregulates CIP2A at the gene transcription level by decreasing the promoter-binding activity of ETS transcription factor (ELK1). In vivo, potency of afatinib against xenograft tumors was also confirmed in nude mice NSCLC models. ${ }^{59}$ Interestingly, ELK1 is overexpressed in lung $\mathrm{SqCC}$ and its overexpression is associated with a poor prognosis. ${ }^{55,59}$ Overall, targeting CIP2A is likely a potential mechanism of afatinib able to limit the progression of lung SqCC. ${ }^{55}$

Previous considerations underline the importance of additional prognostic and predictive data in squamous histology. Among emerging biomarkers, VeriStrat could add useful information: it is a commercially available serum protein test that identifies host patient response to the tumor by measuring acute-phase reactant proteins in the blood and uses matrix-assisted laser desorption ionization time-offlight (MALDI-TOF) mass spectrometry to assign a "good" (VS-G) or "poor" (VS-P) classification. ' In those patients treated with an EGFR TKI, it was evidenced that VS-G 
status was associated with better OS than VS-P status. As a consequence, VeriStrat was thought to be a potential test to predict the clinical outcome of patients with NSCLC after treatment with EGFR TKIs, but different studies indicated that it also has a prognostic role; in fact, the clinical utility of VeriStrat was confirmed by retrospective and prospective studies of different treatment regimens and indications. ${ }^{7}$ Considering first- and second-generation EGFR TKIs, Gadgeel et al, in a retrospective analysis of the Phase III LUX-Lung 8 trial, assessed VeriStrat serum protein test as a predictive marker of differential clinical benefit with afatinib versus erlotinib and the association of VeriStrat status with clinical outcomes irrespective of the EGFR TKI used. Of the 795 patients randomized in LUX-Lung $8, \mathrm{n}=675$ were classified as VS-G: 412 and VS-P: 263. In the VS-G group, OS was significantly longer with afatinib versus erlotinib (HR 0.79, 95\% CI: 0.63-0.98). In the VS-P group, there was no significant difference in OS between the two TKIs (HR 0.90, 0.70-1.16), suggesting that VeriStrat classification may guide treatment decisions in patients with lung SqCC. ${ }^{7}$

\section{Afatinib: safety, tolerability, and patient perspectives}

The safety of afatinib has been frequently a point of discussion: diarrhea was the most common side effect in LUXLung trials, with $17-22 \%$ of patients presenting this AE as a grade $3 .{ }^{42,55}$

Concerning LUX-Lung studies addressed to NSCLC patients or with squamous histology, in LUX-Lung 5, 82\% of patients started on the $40 \mathrm{mg}$ afatinib dose and $27.3 \%$ and $4.5 \%$ of them required one or two afatinib dose reductions, respectively. The most common treatment-related AEs in the combination arm were diarrhea (53.8\%), alopecia (32.6\%), asthenia (27.3\%), decreased appetite (22.0\%), and rash (20.5\%). Serious treatment-related AEs were reported in 11.4 and $3.3 \%$ of patients in the combination and chemotherapy arms, respectively. Dose-reduction rates due to AEs were $32.6 \%$ in the combination group and $11.7 \%$ with chemotherapy, while discontinuation rates due to drug-related AEs were 18.9 and $6.7 \%$ in the two arms, respectively. Among 90 patients with lung SqCC, the most common AEs were asthenia (27.3\%) and diarrhea (18.2\%). Eight (72.7\%) and two (40\%) lung SqCC patients experienced grade 3 AEs with afatinib/ paclitaxel and investigator's choice, respectively, and $27.3 \%$ of patients needed to discontinue afatinib/paclitaxel due to AEs. ${ }^{52}$

In LUX-Lung 8, each group had a similar percentage of patients with AEs of any grade: 390 (99\%) of 392 patients in the afatinib arm versus $385(97 \%)$ of 395 in the erlotinib arm. The severity of AEs (grade $>3$ ) was basically the same in each $\operatorname{arm}(n=224,57 \%$ versus $227,57 \%)$. Grade $>3$ AEs, such as diarrhea (10.4 versus $2.6 \%$ ) and stomatitis (4.1 versus $0 \%$ ), were more frequent with afatinib than erlotinib while treatment-related grade 3 rash or acne was more frequent with erlotinib (10.4\%) than afatinib (5.9\%). The rate of dose reductions due to AEs was higher for the afatinib group compared with the erlotinib group: 27 and 14\%, respectively; treatment discontinuations due to AEs were also higher in the afatinib than in the erlotinib arm: 20 versus $17 \%$, respectively. ${ }^{35}$

Up to $68 \%$ of patients, when questioned, said that they would prefer a therapy that improved disease-related symptoms without prolonging life, as opposed to a therapy that improved survival without symptom benefit. ${ }^{60}$

Given that NSCLC symptoms, including cough, dyspnea, and pain, have a profound impact on health-related quality of life (HR QoL), LUX-Lung 8 also integrated end-points to evaluate comprehensive patient-reported outcomes (PROs). ${ }^{61}$ Patients $(\mathrm{n}=795)$ were randomized $1: 1$ to oral afatinib $(40 \mathrm{mg} /$ day) or erlotinib (150 mg/day). PROs were collected using the European Organization for Research and Treatment of Cancer Quality of Life Questionnaire C30 and its lung cancer-specific module (QLQ-LC13) at the first visit of each treatment course and at the end of treatment. Questionnaires were completed independently by the patients prior to clinical assessment to ensure that responses were an accurate expression of how they felt about their condition without any influence from their treating physician. The percentage of patients who improved during therapy, time to deterioration (TTD), and changes over time were analyzed for prespecified lung cancer-related symptoms, global health status (GHS)/QoL. Questionnaire compliance was high in both arms: 77.3-99\% and 68.7-99\% with afatinib and erlotinib, respectively. Significantly, more patients in the afatinib arm reported improved GHS/QoL and cough compared with the erlotinib arm (36 versus $28 \%, P=0.041$ and 43 versus $35 \% ; P=0.029$, respectively). Afatinib significantly delayed TTD in dyspnea $(P=0.008)$ versus erlotinib, but not cough $(P=0.256)$ or pain $(P=0.869){ }^{61,62}$ However, as already stated before, particularly for afatinib, diarrhea was the most impacting toxicity: in LUX-Lung 8, the frequency of treatment-related grade 3/4 diarrhea was higher with afatinib, although discontinuations because of diarrhea were low for both treatment arms $(4.1 \%$ with afatinib and $1.5 \%$ with erlotinib). In a small sub-study to assess the effect of diarrhea from a patient's perspective, a selected subset of 63 patients (afatinib $n=36$, erlotinib $n=27$ ) consented to provide a detailed diary of the onset, intensity, and duration of diarrhea (any grade) in the first 12 weeks 
of the study. The overall incidence of diarrhea was consistent with that in the main trial population, with 31 (86.1\%) patients in the afatinib arm, and $14(51.8 \%)$ patients in the erlotinib arm, reporting diarrhea as an AE. Seven patients experienced grade 3 diarrhea in the afatinib group; no patients in the sub-study discontinued afatinib treatment because of diarrhea, although seven patients in the afatinib arm had diarrhea-related dose reductions. ${ }^{61}$

\section{Conclusion}

The opportunity to definitively establish EGFR inhibition as a feasible therapeutic strategy in advanced lung SqCC was hampered for a long time by a limited power in early trials, considering that patients with squamous histology usually constitute at best $20-30 \%$ of the study populations. ${ }^{17,18,22}$ The amount of research insight about the EGFR pathway in squamous histology is increasing however, for now, EGFR TKIs may continue to play an important but limited role in the treatment of patients with advanced lung SqCC. This is firstly due to the lack of predictive biomarkers able to select responsive lung SqCC patients to the anti-EGFR approach, and also because of highly active treatments in this setting which inevitably led to uncertainties regarding optimal management and sequence of different options.

Finally, as more treatment opportunities become available for patients, what will be most important is to tailor the different therapeutic alternatives to the patient's own preferences and tolerability, considering the longer lifetime of patients with advanced lung cancer, as well as affordability, especially in the era of increasing health care costs.

\section{Acknowledgment}

The author thanks Silvia Novello for interesting discussions on the subject matter.

\section{Disclosure}

The author reports no conflicts of interest in this work.

\section{References}

1. WHO Statistics.int [webpage on the Internet]. Available from: http:// www.who.int/mediacentre/factsheets/fs297/en/. Accessed July 21, 2017.

2. Travis WD. Pathology of lung cancer. Clin Chest Med. 2011;32:669-692.

3. Vavalà T, Novello S. SC10.04 second-line therapy and beyond in squamous cell NSCLC. J Thorac Oncol. 2017;12(1):S101-S102.

4. Hirsch FR, Varella-Garcia M, Bunn PA, et al. Epidermal growth factor receptor in non-small-cell lung carcinomas: correlation between gene copy number and protein expression and impact on prognosis. J Clin Oncol. 2003;21(20):3798-3807.

5. López-Malpartida AV, Ludeña MD, Varela G, García Pichel J. Differential ErbB receptor expression and intracellular signaling activity in lung adenocarcinomas and squamous cell carcinomas. Lung Cancer. 2009;65(1):25-33.
6. Lo Russo G, Proto C, Garassino MC. Afatinib in the treatment of squamous non-small cell lung cancer: a new frontier or an old mistake? Transl Lung Cancer Res. 2016;5(1):110-114.

7. Gadgeel S, Goss G, Soria JC, et al. Evaluation of the VeriStrat ${ }^{\circledR}$ serum protein test in patients with advanced squamous cell carcinoma of the lung treated with second-line afatinib or erlotinib in the phase III LUXLung 8 study. Lung Cancer. 2017;109:101-108.

8. Ameratunga M, Pavlakis N, Gebski V, Broad A, Khasraw M. Epidermal growth factor receptor-tyrosine kinase inhibitors in advanced squamous cell carcinoma of the lung: a meta-analysis. Asia Pac J Clin Oncol. 2014;10(3):273-278.

9. The Cancer Genome Atlas Research Network. Comprehensive genomic characterization of squamous cell lung cancers. Nature. 2012;489(7417):519-525.

10. Ugocsai K, Mandoky L, Tiszlavicz L, Molnar J. Investigation of HER 2 overexpression in non-small cell lung cancer. Anticancer Res. 2005;25(4):3061-3066.

11. Xu Y, Ding VW, Zhang H, Zhang X, Jablons D, He B. Spotlight on afatinib and its potential in the treatment of squamous cell lung cancer: the evidence so far. Ther Clin Risk Manag. 2016;12:12807-12816.

12. Kan Z, Jaiswal BS, Stinson J, et al. Diverse somatic mutation patterns and pathway alterations in human cancers. Nature. 2010; 466(7308):869-873.

13. Soung YH, Lee JW, Kim SY, et al. Somatic mutations of the ERBB4 kinase domain in human cancers. Int J Cancer. 2006;118(6):1426-1429.

14. Dhanasekaran SM, Alejandro BO, Chen G, et al. Transcriptome metaanalysis of lung cancer reveals recurrent aberrations in NRG1 and Hippo pathway genes. Nat Commun. 2014;5:5893.

15. National Comprehensive Cancer Network [homepage on the Internet]. Head and Neck Cancers - NCCN Guidelines. Version 2.2017-May 8.2017. Available from: http://www.NCCN.org. Accessed July 21, 2017.

16. Pirker R. EGFR-directed monoclonal antibodies in non-small cell lung cancer. Target Oncol. 2013;8(1):47-53.

17. Goss GD, Spaans JN. Epidermal growth factor receptor inhibition in the management of squamous cell carcinoma of the lung. Oncologist. 2016;21(2):205-213.

18. Shepherd FA, Pereira JR, Ciuleanu T, et al. Erlotinib in previously treated non-small-cell lung cancer. $N$ Engl J Med. 2005;353:123-132.

19. Kim ES, Hirsh V, Mok T, et al. Gefitinib versus docetaxel in previously treated non-small-cell lung cancer (INTEREST): a randomised phase III trial. Lancet. 2008;372(9652):1809-1818.

20. Jazieh AR, Al Sudairy R, Abu-Shraie N, Al Suwairi W, Ferwana M, Murad MH. Erlotinib in wild type epidermal growth factor receptor non-small cell lung cancer: a systematic review. Ann Thorac Med. 2013;8(4):204-208.

21. Hanna N, Lilenbaum R, Ansari R, et al. Phase II trial of cetuximab in patients with previously treated non-small cell lung cancer. $J$ Clin Oncol. 2006;24:5253-5258.

22. Thatcher N, Hirsch FR, Luft AV, et al. Necitumumab plus gemcitabine and cisplatin versus gemcitabine and cisplatin alone as first-line therapy in patients with stage IV squamous non-small-cell lung cancer (SQUIRE): an open-label, randomised, controlled phase 3 trial. Lancet Oncol. 2015;16(7):763-774.

23. Pirker R, Pereira JR, von Pawel J, et al. EGFR expression as a predictor of survival for first-line chemotherapy plus cetuximab in patients with advanced non-small-cell lung cancer: analysis of data from the phase 3 FLEX study. Lancet Oncol. 2012;13:33-42.

24. Pirker R, Pereira JR, Szczesna A, et al. Cetuximab plus chemotherapy in patients with advanced non-small-cell lung cancer (FLEX): an openlabel randomised phase III trial. Lancet. 2009;373(9674):1525-1531.

25. Hirsch F, Camidge RD, Kabbinavar F, et al. Biomarker status correlates with clinical benefit: phase 2 study of signal-agent erlotinib or intercalated with carboplatin and paclitaxel in and EGFR biomarker selected NSCLC population. J Thorac Oncol. 2008;3:S267.

26. Gatzemeier U, Pluzanska A, Szczesna A, et al. Phase III study of erlotinib in combination with cisplatin and gemcitabine in advanced non-small-cell lung cancer: the Tarceva Lung Cancer Investigation trial. J Clin Oncol. 2007;25(12):1545-1552. 
27. Giaccone G, Herbst RS, Manegold C, et al. Gefitinib in combination with gemcitabine and cisplatin in advanced non-small-cell lung cancer: a phase III trial - INTACT 1. J Clin Oncol. 2004;22(5):777-784.

28. Reck M, Rodríguez-Abreu D, Robinson AG. Pembrolizumab versus chemotherapy for PD-L1 positive non-small-cell lung cancer. $N$ Engl J Med. 2016;375:1823-1833.

29. FDA [webpage on the Internet]. FDA Approves Keytruda for Advanced Non-Small Cell Lung Cancer. Available from: http://www.fda.gov/NewsEvents/Newsroom/PressAnnouncements/ucm465444.htm. Accessed August 21, 2017.

30. Carbone DP, Reck M, Paz-Ares L, et al. First-line nivolumab in stage IV or recurrent non-small-cell lung cancer. NEnglJ Med.2017;376:2415-2426.

31. Dai Q, Ling YH, Lia M, et al. Enhanced sensitivity to the HER1/ epidermal growth factor receptor tyrosine kinase inhibitor erlotinib hydrochloride in chemotherapy-resistant tumor cell lines. Clin Cancer Res. 2005;11(4):1572-1578.

32. Cappuzzo F, Ciuleanu T, Stelmakh L, et al. Erlotinib as maintenance treatment in advanced non-small-cell lung cancer: a multicentre, randomised, placebo-controlled phase 3 study. Lancet Oncol. 2010;11(6):521-529.

33. Garon EB, Ciuleanu TE, Arrieta O, et al. Ramucirumab plus docetaxel versus placebo plus docetaxel for second-line treatment of stage IV non-small-cell lung cancer after disease progression on platinum-based therapy (REVEL): a multicentre, double-blind, randomised phase 3 trial. Lancet. 2014;384:665-673.

34. Brahmer J, Reckamp KL, Baas P, et al. Nivolumab versus docetaxel in advanced squamous-cell non-small-cell lung cancer. $N$ Engl $J$ Med. 2015;373(2):123-135.

35. Soria JC, Felip E, Cobo M, et al. Afatinib versus erlotinib as secondline treatment of patients with advanced squamous cell carcinoma of the lung (LUX-Lung 8): an open-label randomised controlled phase 3 trial. Lancet Oncol. 2015;16:897-907.

36. Melosky B, Chu Q, Juergens R, Leighl N, McLeod D, Hirsh V. Pointed progress in second-line advanced non-small-cell lung cancer: the rapidly evolving field of checkpoint inhibition. J Clin Oncol. 2016;34(14):1676-1688.

37. Le Ang Y, Lim JSJ, Soo RA. Profile of nivolumab in the treatment of metastatic squamous non-small-cell lung cancer. Onco Targets Ther. 2016;9:3187-3195.

38. Herbst RS, Baas P, Kim DW, et al. Pembrolizumab versus docetaxel for previously treated, PD-L1-positive, advanced non-small-cell lung cancer (KEYNOTE-010): a randomised controlled trial. Lancet. 2016;387(10027):15401550.

39. Rittmeyer A, Barlesi F, Waterkamp D, et al. Atezolizumab versus docetaxel in patients with previously treated non-small-cell lung cancer (OAK): a phase 3, open-label, multicentre randomised controlled trial. Lancet. 2017;389(10066):255-265.

40. Solca F, Dahl G, Zoephel A, et al. Target binding properties and cellular activity of afatinib (BIBW 2992), an irreversible ErbB family blocker. $J$ Pharmacol Exp Ther. 2012;343(2):342-350.

41. Hirsh V. Next-generation covalent irreversible kinase inhibitors in NSCLC: focus on afatinib. BioDrugs. 2015;29(3):167-183.

42. Chen X, Zhu Q, Zhu L, et al. Clinical perspective of afatinib in nonsmall cell lung cancer. Lung Cancer. 2013;81(2):155-161.

43. Miller VA, Hirsh V, Cadranel J, et al. Afatinib versus placebo for patients with advanced, metastatic non-small-cell lung cancer after failure of erlotinib, gefitinib, or both, and one or two lines of chemotherapy (LUX-Lung 1): a phase 2b/3 randomised trial. Lancet Oncol. 2012;13(5):528-538.

44. Yang JC, Shih JY, Su WC, et al. Afatinib for patients with lung adenocarcinoma and epidermal growth factor receptor mutations (LUX-Lung 2): a phase 2 trial. Lancet Oncol. 2012;13(5):539-548.

45. Sequist LV, Yang JCH, Yamamoto N, et al. Phase III study of afatinib or cisplatin plus pemetrexed in patients with metastatic lung adenocarcinoma with EGFR mutations. J Clin Oncol. 2013;31(27):3327-3334.
46. Wu YL, Zhou C, Hu CP, et al. Afatinib versus cisplatin plus gemcitabine for first-line treatment of Asian patients with advanced non-small-cell lung cancer harbouring EGFR mutations (LUX-Lung 6): an open-label, randomised phase 3 trial. Lancet Oncol. 2014;15(2):213-222.

47. Katakami N, Atagi S, Goto K, et al. LUX-Lung 4: a phase II trial of afatinib in patients with advanced non-small-cell lung cancer who progressed during prior treatment with erlotinib, gefitinib, or both. J Clin Oncol. 2013;31(27):3335-3341.

48. Schuler M, Yang JCH, Park K, et al. Afatinib beyond progression in patients with non-small-cell lung cancer following chemotherapy, erlotinib/gefitinib and afatinib: phase III randomized LUX-Lung 5 trial. Ann Oncol. 2016;27:417-423.

49. Park K, Kim J, Schuler M, et al. Afatinib (A) followed by A+ paclitaxel (P) or investigator's choice of single-agent chemotherapy (IC) in patients (pts) with advanced squamous cell carcinoma (SCC) of the lung: subgroup analysis of LUX-Lung 5 (LL5). Ann Oncol. 2014;25(suppl 4): iv443-iv444.

50. Park K, Tan EH, O'Byrne K, et al. Afatinib versus gefitinib as first-line treatment of patients with EGFR mutation-positive non-small-cell lung cancer (LUX-Lung 7): a phase 2B, open-label, randomised controlled trial. Lancet Oncol. 2016;17(5):577-589.

51. Paz-Ares L, Tan EH, O'Byrne K, et al. Afatinib versus gefitinib in patients with EGFR mutation-positive advanced non-small-cell lung cancer: overall survival data from the phase IIb LUX-Lung 7 trial. Ann Oncol. 2017;28(2):270-277.

52. boehringer-ingelheim.com [webpage on the Internet]. FDA Approves Gilotrif ${ }^{\circledR}$ (afatinib) as New Oral Treatment Option for Patients with Squamous Cell Carcinoma of the Lung. 2016. Available from: http://us.boehringer-ingelheim.com/news_events/press_releases/ press_release_archive/2016/4-15-2016-fda-approves-gilotrif-afatiniboral-treatment-option-patients-squamous-cell-carcinoma-lung.html. Accessed June 15, 2017.

53. Garassino MC, Martelli O, Broggini M, et al. Erlotinib versus docetaxel as second-line treatment of patients with advanced non-small-cell lung cancer and wild-type EGFR tumours (TAILOR): a randomised controlled trial. Lancet Oncol. 2013;14(10):981-988.

54. Kawaguchi T, Ando M, Asami K, et al. Randomized phase III trial of erlotinib versus docetaxel as second- or third line therapy in patients with advanced non-small-cell lung cancer: Docetaxel and Erlotinib Lung Cancer Trial (DELTA). J Clin Oncol. 2014;32(18): 1902-1908

55. Tan WL, Quan-Sing N. The continuing role of epidermal growth factor receptor tyrosine kinase inhibitors in advanced squamous cell carcinoma of the lung. Transl Lung Cancer Res. 2016;5(1):106-109.

56. De Greve J, Moran T, Graas MP, et al. Phase II study of afatinib, an irreversible ErbB family blocker, in demographically and genotypically defined lung adenocarcinoma. Lung Cancer. 2015;88(1):63-69.

57. Cappuzzo F, Finocchiaro G, Grossi F, et al. Phase II study of afatinib, an irreversible ErbB family blocker, in EGFR FISH-positive non-small-cell lung cancer. J Thorac Oncol. 2015;10(4):665-672.

58. Nelson V, Ziehr J, Agulnik M, Johnson M. Afatinib: emerging nextgeneration tyrosine kinase inhibitor for NSCLC. Onco Targets Ther. 2013;6:135-143.

59. Chao TT, Wang CY, Chen YL, et al. Afatinib induces apoptosis in NSCLC without EGFR mutation through Elk-1-mediated suppression of CIP2A. Oncotarget. 2015;6(4):2164-2179.

60. Hirsh V. Is the evaluation of quality of life in NSCLC trials important? Are the results to be trusted? Front Oncol. 2014;4:173.

61. Felip E, Hirsh V, Popat S, et al. Symptom and quality of life improvement in LUX-lung 8, an open-label phase III study of second-line afatinib versus erlotinib in patients with advanced squamous cell carcinoma of the lung after first-line platinum-based chemotherapy. Clin Lung Cancer. Epub 2017 June 23.

62. Hirsh V. New developments in the treatment of advanced squamous cell lung cancer: focus on afatinib. Onco Targets Ther. 2017;10:2513-2526. 
Clinical Pharmacology: Advances and Applications is an international, Visit http://www.dovepress.com/testimonials.php to read real quotes peer-reviewed, open access journal publishing original research, reports, from published authors.

reviews and commentaries on all areas of drug experience in humans.

The manuscript management system is completely online and includes a very quick and fair peer-review system, which is all easy to use.

Submit your manuscript here: https://www.dovepress.com/clinical-pharmacology-advances-and-applications-journal 